TITLE:

\title{
Note: Low energy inverse photoemission spectroscopy apparatus.
}

$\operatorname{AUTHOR}(S)$ :

Yoshida, Hiroyuki

CITATION:

Yoshida, Hiroyuki. Note: Low energy inverse photoemission

spectroscopy apparatus.. The Review of scientific instruments 2014, 85(1): 016101.

ISSUE DATE:

2014-01-08

URL:

http://hdl.handle.net/2433/182927

RIGHT:

(C) 2014 AIP Publishing LLC 




Note: Low energy inverse photoemission spectroscopy apparatus

Hiroyuki Yoshida

Citation: Review of Scientific Instruments 85, 016101 (2014); doi: 10.1063/1.4860055

View online: http://dx.doi.org/10.1063/1.4860055

View Table of Contents: http://scitation.aip.org/content/aip/journal/rsi/85/1?ver=pdfcov

Published by the AIP Publishing

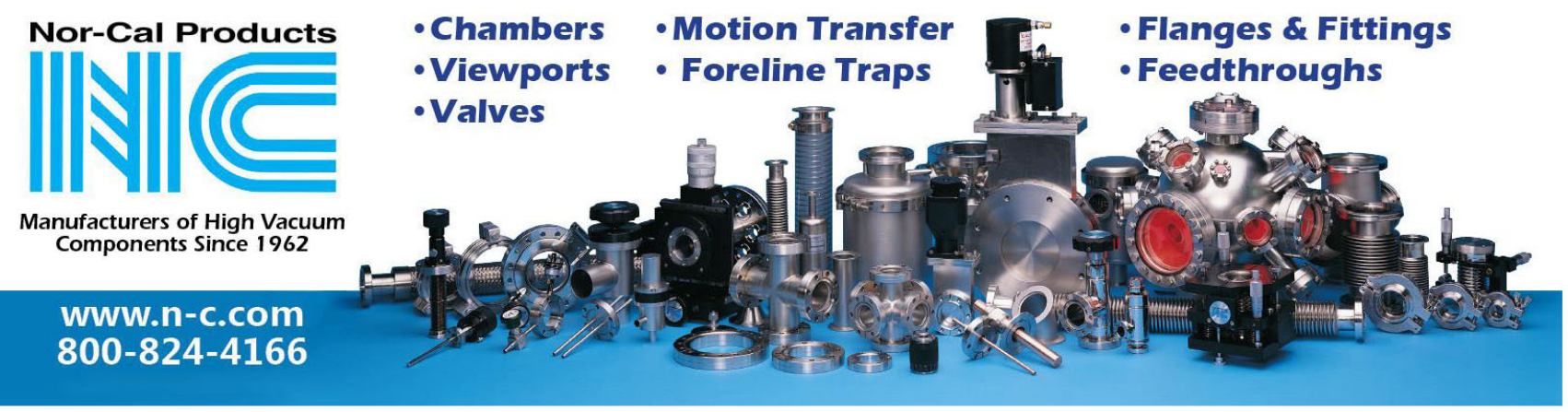




\title{
Note: Low energy inverse photoemission spectroscopy apparatus
}

\author{
Hiroyuki Yoshida ${ }^{\text {a) }}$ \\ Institute for Chemical Research, Kyoto University, Uji, Kyoto 611-0011, Japan
}

(Received 9 September 2013; accepted 16 December 2013; published online 8 January 2014)

\begin{abstract}
An apparatus for the low-energy inverse photoemission spectroscopy is described. In this technique, low energy electron having kinetic energy below $4 \mathrm{eV}$ is incident to the sample and detect the emitted photons in the near ultraviolet range (below $5 \mathrm{eV}$, longer than $250 \mathrm{~nm}$ ) to investigate the unoccupied states of the solid materials. Compared with the prototype apparatus reported previously [H. Yoshida, Chem. Phys. Lett. 539-540, 180-185 (2012)], the collection efficiency of photons is improved by a factor of four and practically any conductive substrates can be used. The overall resolution is $0.27 \mathrm{eV}$. (C) 2014 AIP Publishing LLC. [http://dx.doi.org/10.1063/1.4860055]
\end{abstract}

Inverse photoemission spectroscopy (IPES) is an experimental method to examine the unoccupied states of solid materials. ${ }^{1,2}$ In IPES, mono-energetic electrons are incident on a sample surface and the emitted photons as a result of the electron transition to a particular unoccupied state are detected. IPES can be regarded as the inversion of photoemission spectroscopy (PES) which examines the density of occupied states. However, IPES is far less widely used than PES because of the low cross section of the IPES process. ${ }^{3,4}$ IPES became more widespread after the development of high sensitivity bandpass photon detector in the late 1970s by Dude. ${ }^{5}$ Since then considerable effort has been devoted to improving the energy resolution. ${ }^{6-15}$ However, the bandpass energy and energy resolution are inherently limited by the properties of the materials used. The resolution of practically useful apparatus is limited to $0.4 \mathrm{eV} \cdot{ }^{12-15}$ Another large drawback of IPES is sample damage when it is applied to organic materials. ${ }^{16}$

Recently, we have demonstrated low-energy inverse photoemission spectroscopy (LEIPS) for the first time. ${ }^{17}$ The electron kinetic energy ranges between 0 and $4 \mathrm{eV}$ and the emitted photons in the near ultraviolet (NUV; wavelength longer than $250 \mathrm{~nm}$ ) range are detected. Compared with the vacuum ultraviolet light typically detected in the conventional IPES, the NUV photons can be analyzed at the higher resolution without losing the sensitivity. We have demonstrated LEIPS using the multilayer dielectric interference filters (the resolution between 0.1 and $0.25 \mathrm{eV})^{17}$ or the high-resolution grating spectrometer (better than $0.1 \mathrm{eV}$ ). ${ }^{18}$ Since the kinetic energy of electron is below the damage threshold of most of organic materials, ${ }^{19}$ the irradiation damage to the organic samples can be significantly reduced. So far, the electron affinities of a n-type polymer, $\mathrm{P}(\mathrm{NDI} 2 \mathrm{OD}-\mathrm{T} 2),{ }^{20}$ copper phthalocyanine $(\mathrm{CuPc}),{ }^{17}$ and pentacene ${ }^{21}$ have been precisely determined.

In this Note, we describe an experimental apparatus for LEIPS. Compared with the prototypical apparatus reported before, ${ }^{17}$ two major improvements have been made. First, the photon detector was installed on the same side of the electron

\footnotetext{
a)Email: yoshida@e.kuicr.kyoto-u.ac.jp. TEL: +81-774-38-3083. FAX: +81-774-38-3084.
}

source. This enabled us to use any conductive materials such as metal and semiconductor. Second, the focusing optics were installed in the vacuum chamber. This improvement increases the collection efficiency of photons by a factor of four.

The experimental apparatus consists of the two vacuum chambers, one for the sample preparation (not shown) and the other for LEIPS measurement (Fig. 1), which are evacuated to lower than $1 \times 10^{-7} \mathrm{~Pa}$. In the LEIPS chamber, an electron beam is incident to a sample film. The sample current is measured using an Advantest 8240 electrometer. The emitted photons are collected and focused into a photon detector using a quartz lens. The lens was installed as close as the sample specimen resulting in the acceptance angle of $0.4 \mathrm{sr}$. The collected photons are selected by an optical bandpass filter (BrightLine, Semrock Inc.) and detected by a photomultiplier tube (R585, Hamamatsu Photonics K.K). The photon intensity is recorded as a function of electron kinetic energy and normalized by the incident electron current to yield a LEIPS spectrum.

The overall resolution of IPES apparatus is assessed from a spectrum of the Fermi edge of an Ag film. Figure 2(a) shows the spectrum taken with the bandpass filter of $254 \mathrm{~nm}$ (center energy of $4.88 \mathrm{eV}$ with $0.15 \mathrm{eV}$ in a full width at the half maximum, FWHM). The spectrum closely follows the error function with an FWHM of $0.27 \mathrm{eV}^{22}$ The instrumental function is evaluated from the first derivative of the spectrum around the Fermi level ${ }^{17}$ and can be approximated by the Gaussian function as shown in Fig. 2(b). The determined overall resolution is $0.27 \pm 0.01 \mathrm{eV}$ in FWHM. Though the two procedures are mathematically equivalent, the first method does not require the derivative and a spectrum with lower $\mathrm{S} / \mathrm{N}$ ratio can be analyzed. The overall resolution thus derived is improved from the previous value $0.33 \pm 0.03 \mathrm{eV}^{17}$ mainly because the resolution of the bandpass filter is improved from $0.20 \mathrm{eV}$ to $0.15 \mathrm{eV}$.

Using this apparatus, a thin film of zinc phthalocyanine $(\mathrm{ZnPc})$ was examined. Figure 3 shows the IPES spectra of a 10 -nm thick ZnPc film. The spectra measured at the different photon energies, 254, 285, and $335 \mathrm{~nm}$ show the same spectral features suggesting that the spectra certainly reflect the density of unoccupied states of the $\mathrm{ZnPc}$ film. The spectra are also consistent with the earlier reports. ${ }^{23,24}$ 
Hiroyuki Yoshida

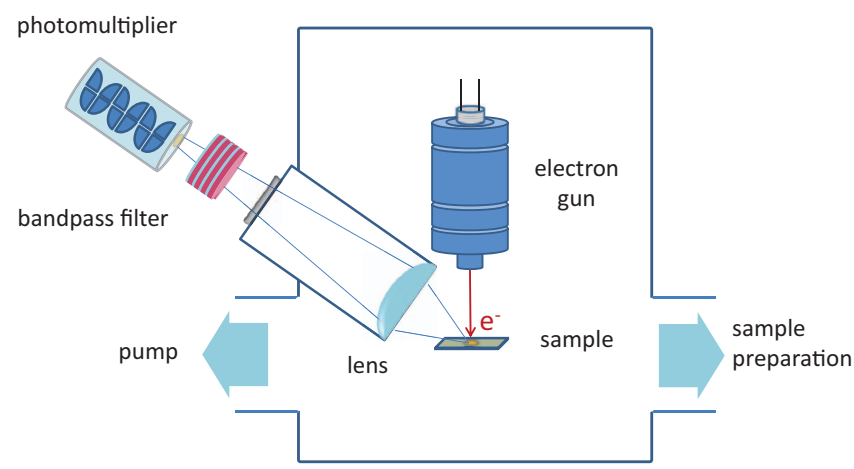

FIG. 1. Schematic diagram of the LEIPS apparatus.

The intensity of the spectra can be compared with those of $\mathrm{CuPc}$ in the previous report ${ }^{17}$ because the IPES spectra around the energy gap are mainly contributed from the organic ligand rather than the central metal. ${ }^{23}$ The intensities of Fig. 3 are one order of magnitude higher than the previous ones (Fig. 4 of Ref. 17) if the throughput of the filters is taken into account. This increase of the signal intensity can be understood from the two reasons. First, the collection efficiency of the photon is improved by a factor of four. Second, the photon detector is installed in the same side of the electron gun so that the photons are not absorbed by the sample and substrate; the transmittance of 20-nm-thick ITO used in Ref. 17 as a substrate is only $40 \%$ in $250-300 \mathrm{~nm}$.

In summary, an experimental apparatus for the LEIPS is described. Compared with the prototypical apparatus for LEIPS,${ }^{17}$ the solid angle for the photon collection is increased fourfold by placing the focusing lens in the vacuum. In addi-



FIG. 2. (a) The LEIPS spectrum around the Fermi level of Ag thin film. (b) The first derivative of the experimental spectrum showing the instrumental function. ${ }^{17}$ The dots show the experimental data and the solid lines represent the best-fit results of the error function (a) and the Gaussian function (b).

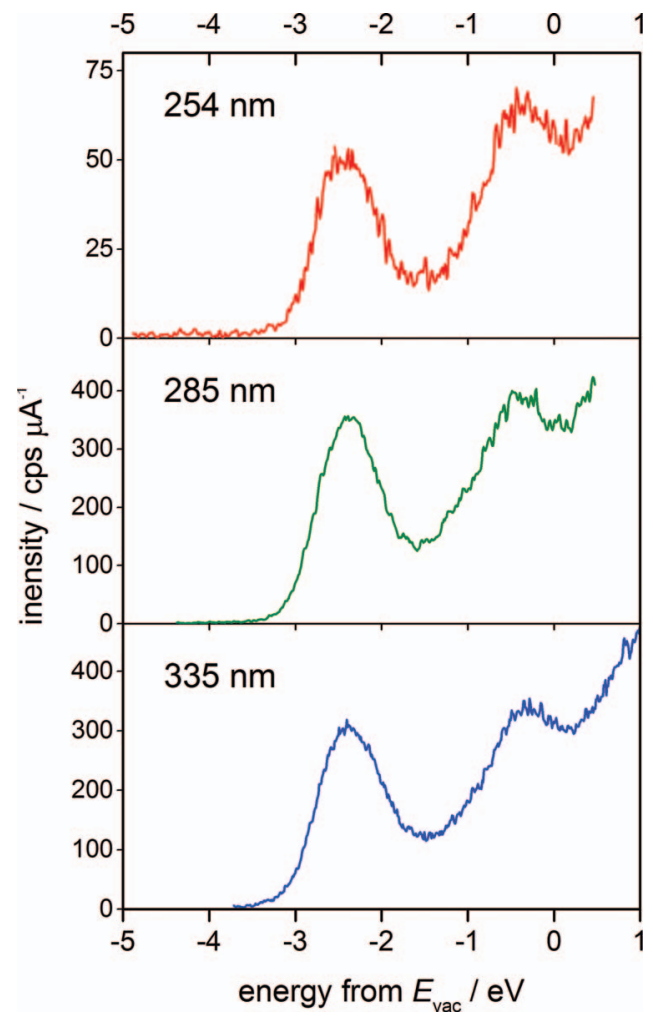

FIG. 3. IPES spectra of zinc phthalocyanine ( $\mathrm{ZnPc})$ using the bandpass filters with different center wavelength.

tion, the photon detector is installed facing the sample surface. This allows us to use opaque substrates in NUV range (any materials that have electrical conductivity). The photon signals are not lost by the absorption in the sample and substrate together with the improved collection efficiency, the signal intensity is increased by one order of magnitude. The overall resolution, estimated from the Fermi edge of $\mathrm{Ag}$ film, is $0.27 \mathrm{eV}$.

The author thanks Mr. Kunihiro Aoshima of ADCAP Vacuum Technology Inc. for technical assistance. Dr. Richard Murdey and Prof. Naoki Sato of Kyoto University are acknowledged for their continual encouragement. This work was supported by JST, PRESTO.

${ }^{1}$ P. D. Johnson and S. L. Hulbert, Rev. Sci. Instrum. 61, 2277 (1990).

${ }^{2}$ J. C. Fuggle and J. E. Inglesfield, Top. Appl. Phys. 69, 1 (1992).

${ }^{3}$ J. B. Pendry, Phys. Rev. Lett. 45, 1356 (1980).

${ }^{4}$ J. B. Pendry, J. Phys. C: Solid State Phys. 14, 1381 (1981).

${ }^{5}$ V. Dose, Appl. Phys. 14, 117 (1977).

${ }^{6}$ N. Babbe, W. Drube, I. Schafer, and M. Skibowski, J. Phys. E 18, 158 (1985).

${ }^{7}$ V. Dose, T. Fauster, and R. Schneider, Appl. Phys. A 40, 203 (1986).

${ }^{8}$ D. Funnemann and H. Merz, J. Phys. E 19, 554 (1986).

${ }^{9}$ K. Yokoyama, K. Nishihara, K. Mimura, Y. Hari, M. Taniguchi, Y. Ueda, and M. Fujisawa, Rev. Sci. Instrum. 64, 87 (1993).

${ }^{10}$ F. Schedin, G. Thornton, and R. I. G. Uhrberg, Rev. Sci. Instrum. 68, 41 (1997).

${ }^{11}$ J. A. Lipton-Duffin, A. G. Mark, and A. B. McLean, Rev. Sci. Instrum. 73, 3149 (2002).

${ }^{12}$ M. Budke, V. Renken, H. Liebl, G. Rangelov, and M. Donath, Rev. Sci. Instrum. 78, 083903 (2007).

${ }^{13}$ R. Stiepel, R. Ostendorf, C. Benesch, and H. Zacharias, Rev. Sci. Instrum. 76, 063109 (2005).

${ }^{14}$ M. Maniraj, S. W. D’Souza, J. Nayak, A. Rai, S. Singh, B. N. R. Sekhar, and S. R. Barman, Rev. Sci. Instrum. 82, 093901 (2011). 
${ }^{15}$ M. Maniraj, B. N. R. Sekhar, and S. R. Barman, Rev. Sci. Instrum. 83, 046107 (2012).

${ }^{16}$ K. Tsutsumi, H. Yoshida, and N. Sato, Chem. Phys. Lett. 361, 367 (2002).

${ }^{17}$ H. Yoshida, Chem. Phys. Lett. 539-540, 180 (2012).

${ }^{18}$ H. Yoshida, Rev. Sci. Instrum. 84, 103901 (2013).

${ }^{19}$ B. Boudaiffa, P. Cloutier, D. Hunting, M. A. Huels, and L. Sanche, Science 287, 1658 (2000).

${ }^{20}$ S. Fabiano, H. Yoshida, Z. H. Chen, A. Facchetti, and M. A. Loi, ACS Appl. Mater. Interfaces 5, 4417 (2013).
${ }^{21}$ W. Han, H. Yoshida, N. Ueno, and S. Kera, Appl. Phys. Lett. 103, 123303 (2013).

${ }^{22}$ The error function is integral of the Gaussian function. Hence, the fitting the error function to the original spectrum is mathematically equivalent to fitting the Gaussian function to the first derivative of the spectrum.

${ }^{23}$ H. Yoshida, K. Tsutsumi, and N. Sato, J. Electron Spectrosc. Relat. Phenom. 121, 83 (2001).

${ }^{24}$ W. Y. Gao and A. Kahn, Org. Electron. 3, 53 (2002). 